\title{
Longitudinal coupling impedance of a small hole in a coaxial liner near the cutoff frequencies
}

\author{
Alexei V. Fedotov and Robert L. Gluckstern \\ Physics Department, University of Maryland, College Park, Maryland 20742
}

(Received 14 May 1998; published 19 June 1998)

\begin{abstract}
We recently developed a general analysis for an azimuthally asymmetric rectangular slot in the inner conductor of a coaxial liner, which allowed us to investigate the coupling impedance numerically. In the present paper we obtain analytic expressions for a small hole of arbitrary shape. Specifically, we go beyond the quasistatic (Bethe) approximation to explore and understand the structure of the impedance in the frequency region near the cutoffs of the inner beam pipe and outer coaxial structure. Finally, we extend our analytic analysis to a hole in a wall of finite thickness. [S1098-4402(98)00005-6]
\end{abstract}

PACS numbers: 29.27.Bd, 41.20.-q

\section{INTRODUCTION}

Beam pipes in high-energy superconducting colliders require a shielding tube (liner) with pumping slots to screen cold chamber walls from synchrotron radiation. The pumping slots in the liner are the chamber discontinuities, and electromagnetic fields diffracted by them can affect beam stability. This beam-chamber interaction can be described in terms of the coupling impedance.

In an earlier work, Gluckstern and Neri [1] analyzed the impedance of a small azimuthally symmetric pillbox in a beam pipe at frequencies of the same order as the cutoffs of the $\mathrm{TM}_{0 n}$ modes in the pipe. They found that the admittance could be written as the sum of a term depending primarily on the pillbox width and thickness, and a term depending primarily on the pipe radius. In fact, the broad resonance used frequently by others to describe the behavior near cutoffs was shown to be due to a change of sign of the imaginary part of the admittance.

We recently constructed a variational form for the impedance of a rectangular hole in the wall of a coaxial liner [2]. Our analysis allowed us to study numerically the frequency dependence of the coupling impedance of a transverse rectangular slot, small square hole [2], and a longitudinal rectangular slot, including the resonances due to the slot length [3]. However, it is possible to obtain an approximate analytic expression, analogous to that obtained in [1], for a small hole of arbitrary shape which would allow us to understand the structure of the impedance in the frequency region near the cutoff of the beam pipe. In the case of a narrow pillbox the dominant contribution comes from the magnetic portion of the problem; therefore, in [1], only the magnetic part was considered. In the present paper we extend the analysis to the azimuthally asymmetric problem of a hole in the wall of a coaxial liner. We also consider both the electric and magnetic portions of the problem. Finally, we extend our analysis to a hole in a wall of finite thickness.

\section{THE LONGITUDINAL COUPLING IMPEDANCE}

The source fields in the frequency domain generated by the driving current

$$
J_{z}(x, y, z ; k)=I_{0} \delta(x) \delta(y) e^{-j k z}
$$

are the following:

$$
\begin{gathered}
E_{r}^{(s)}(r, z ; k)=Z_{0} H_{\theta}^{(s)}(r, z ; k)=\frac{Z_{0} I_{0}}{2 \pi r} e^{-j k z}, \\
E_{z}^{(s)}(r, z ; k)=0,
\end{gathered}
$$

where $Z_{0}=120 \pi[\Omega], k=\omega / c$. The definition of the frequency dependent longitudinal coupling impedance of any obstacle can be taken to be

$$
Z_{\|}=\frac{-1}{I_{0}} \int_{-\infty}^{\infty} d z e^{j k z} E_{z}(0, \theta, z ; k),
$$

where $E_{z}(r, \theta, z ; k)$ is the axial electric field in the frequency domain, with frequency dependence $\exp (j \omega t)$, where $\omega=k c$. This expression can be rewritten as

$$
\frac{Z_{\|}(k)}{Z_{0}}=-\frac{1}{2 \pi a Z_{0} I_{0}} \int d S E_{z}(a, \theta, z ; k) e^{j k z},
$$

where the surface integral is over only the hole, since $E_{z}$ vanishes on the liner wall.

Since the driving current on axis is proportional to $\exp (-j k z)$, the problem is simplified by obtaining results for an even driving current $\cos k z$ and an odd driving current $-j \sin k z$ separately. In the even problem $E_{z}$, $H_{r}$, and $H_{\theta}$ are even in $z$, while in the odd problem $E_{z}$, $H_{r}$, and $H_{\theta}$ are odd in $z$ (where $z=0$ is chosen to be the center of the hole). In any case $E_{z}, E_{r}$, and $H_{\theta}$ are always even in $\theta$, and $H_{z}, H_{r}$, and $E_{\theta}$ are always odd in $\theta$. We use the superscript $(e)$ for the even problem and the superscript $(o)$ for the odd problem. The field matching is performed at the radius of the inner conductor (liner) in the opening. We call the region inside the inner conductor 
$r \leq a$ the "pipe region" and the region outside the inner conductor $a \leq r \leq b$ the "coaxial region." Note that we consider the inside and outside surfaces of the liner both to be at $r=a$, since we neglect the thickness of the liner compared to the wavelength and to the dimensions of the hole. The technique consists of expanding fields in both regions into a complete set of functions. At the common interface the fields have to be matched yielding equations for the expansion coefficients.

\section{GENERAL BACKGROUND}

In the pipe region the fields are given by the source fields plus a general solution of the Maxwell equations for the cylindrical waveguide. In the coaxial region we have a general solution of the Maxwell equations for the coaxial waveguide. Because of the asymmetry of the problem we need to consider both the TM and TE modes. We first consider the even portion of the problem.

For the TM portion of the modes we have

where

$$
E_{z}^{(e)}(r, \theta, z)=\int d q \cos q z \phi^{(e)}(r, \theta),
$$

$$
\phi^{(e)}(r, \theta)=\sum_{n} \cos n \theta A_{n}^{(e)}(q)\left[\frac{J_{n}(\kappa r)}{J_{n}(\kappa a)}, \frac{F_{n}(\kappa r)}{F_{n}(\kappa a)}\right],
$$

with $\kappa$, defined by $\kappa^{2}=k^{2}-q^{2}$, being the radial propagation constant. Here we use the notation where the first part in square brackets corresponds to the pipe region $r \leq a$, and the second part corresponds to the coaxial region $a \leq r \leq b$, with the function $F_{n}$ being the solution of the Maxwell equations for the coaxial region for the TM modes $\left[F_{n}(u)=Y_{n}(u) J_{n}(\kappa b)-J_{n}(u) Y_{n}(\kappa b)\right]$. The coefficient $A_{n}^{(e)}(q)$ is the same for both $r<a$ and $r>a$, since $E_{z}^{(e)}$ is continuous at $r=a$ within the hole and on both sides of the liner surface, where $E_{z}^{(e)}=0$.

For the TE portion of the modes we similarly have

$$
Z_{0} H_{z}^{(e)}(r, \theta, z)=\int d q \sin q z \psi^{(e)}(r, \theta),
$$

where

$$
\psi^{(e)}(r, \theta)=-\sum_{n} \sin n \theta B_{n}^{(e)}\left[\frac{J_{n}(\kappa r)}{J_{n}^{\prime}(\kappa a)}, \frac{G_{n}(\kappa r)}{G_{n}^{\prime}(\kappa a)}\right],
$$

with the function $G_{n}(\kappa r)$ being the solution of the Maxwell equations for the coaxial region for the TE modes $\left[G_{n}(u)=Y_{n}(u) J_{n}^{\prime}(\kappa b)-J_{n}(u) Y_{n}^{\prime}(\kappa b)\right]$. The other TE and TM field components can be easily obtained using Maxwell equations [2]. For the expansion coefficients we obtain [2]

$$
A_{n}^{(e)}(q)=\frac{1}{4 \pi^{2} a} \int d S \cos q z \cos n \theta E_{z}^{(e)}(a, \theta, z)
$$

by matching $E_{z}$ at $r=a$, and

$$
B_{n}^{(e)}(q)=j \frac{\kappa}{k} \frac{1}{4 \pi^{2} a} \int d S\left[\sin q z \sin n \theta E_{\theta}^{(e)}(a, \theta, z)-\frac{q n}{a \kappa^{2}} \cos q z \cos n \theta E_{z}^{(e)}(a, \theta, z)\right]
$$

by matching $E_{\theta}$ at $r=a$, which also must vanish on the metallic surface.

For the odd portion of the problem, $E_{z}^{(o)}$ is an odd function in $z$. We perform expansion for the fields similar to those for the even portion of the problem. In the expressions for the fields in Eqs. (6)-(9) we replace $\cos q z$ by $-j \sin q z$ and $\sin q z$ by $j \cos q z$. In the expressions for the coefficients in Eqs. (10) and (11) we replace $\cos q z$ by $j \sin q z$ and $\sin q z$ by $-j \cos q z$.

\section{THIN WALL ANALYSIS}

\section{A. Odd part}

We now assume that the hole dimensions are small compared to the wavelength, and we can use the quasistatic solutions for the field components in the vicinity of the hole. The odd part of the impedance is then given by

$$
\begin{aligned}
\frac{Z_{\|}^{(o)}(k)}{Z_{0}} & =-\frac{j}{2 \pi a Z_{0} I_{0}} \int d S \sin k z E_{z}^{(o)}(a, \theta, z ; k) \\
& =-\frac{j k}{2 \pi a Z_{0} I_{0}} \int \Phi d S
\end{aligned}
$$

where the electrostatic approximation $E_{z}=-\frac{\partial \Phi}{\partial z}$ was used. We rewrite the above integral as

$$
\begin{aligned}
& \int \Phi d S= \frac{\chi}{2}\left[E_{r}^{(o)}(a-)-E_{r}^{(o)}(a+)\right] \\
&=\frac{\chi}{2}\left[\frac{Z_{0} I_{0}}{2 \pi a} \cos k z-j a \sum_{n} \int d q q A_{n}^{(o)} P_{n}(q)\right. \\
&\left.\quad-k \sum_{n} \int d q \frac{n}{\kappa} B_{n}^{(o)} Q_{n}(q)\right],
\end{aligned}
$$

where $\chi$ is the electric polarizability of a hole, and the functions $P_{n}(q)$ and $Q_{n}(q)$ are given by the following expressions:

$$
\begin{aligned}
& P_{n}(q)=\left[\frac{J_{n}^{\prime}(\kappa a)}{\kappa a J_{n}(\kappa a)}-\frac{F_{n}^{\prime}(\kappa a)}{\kappa a F_{n}(\kappa a)}\right], \\
& Q_{n}(q)=\left[\frac{J_{n}(\kappa a)}{\kappa a J_{n}^{\prime}(\kappa a)}-\frac{G_{n}(\kappa a)}{\kappa a G_{n}^{\prime}(\kappa a)}\right] .
\end{aligned}
$$

In the small hole approximation for the expansion coefficients we obtain

$$
A_{n}^{(o)}=\frac{j q}{4 \pi^{2} a} \int \Phi d S=\frac{Z_{0} I_{0}}{16 \pi^{3} a^{2}} j q \chi
$$




$$
B_{n}^{(o)}=\frac{Z_{0} I_{0}}{16 \pi^{3} a^{3}} \frac{n k}{\kappa} \chi .
$$

For the odd part of the impedance we then have

$$
\frac{Z_{\|}^{(o)}}{Z_{0}}=-\frac{j k}{8 \pi^{2} a^{2}} \chi\left(1+\frac{\chi}{8 \pi^{2}} W\right),
$$

where $W$ is defined as

$$
W=\sum_{n}\left\{\int d q\left[q^{2} P_{n}(q)-\frac{n^{2} k^{2}}{\kappa^{2} a^{2}} Q_{n}(q)\right]\right\} .
$$

For a small hole, the term proportional to $\chi W$ will be small compared to 1 , and we can write an expression for the admittance as

$$
Z_{0} Y_{\|}^{(o)}=\frac{j 8 \pi^{2} a^{2}}{k \chi}-\frac{j a^{2}}{k} W .
$$

\section{B. Even part}

We now have

$$
\int d S E_{z}(a, \theta, z)=j k \psi_{\theta} \frac{H_{\theta+}-H_{\theta-}}{2},
$$

where $\psi_{\theta}$ is the transverse magnetic susceptibility of a hole. Similarly to the odd part, for the expansion coefficients we obtain

$$
\begin{aligned}
A_{n}^{(e)} & =\frac{1}{4 \pi^{2} a} \int d S \cos q z \cos n \theta E_{z}^{(e)}(a, \theta, z) \\
& =\frac{-j k \psi_{\theta}}{2}\left(H_{\theta-}-H_{\theta+}\right) \frac{Z_{0}}{4 \pi^{2} a} \\
& =-\frac{j k \psi_{\theta}}{16 \pi^{3} a^{2}} Z_{0} I_{0}, \\
B_{n}^{(e)}= & \frac{j \kappa}{4 \pi^{2} a k} \int d S \sin q z \sin n \theta E_{\theta}^{(e)}(a, \theta, z) \\
& -\frac{1}{4 \pi^{2} a} \frac{j q n}{k a \kappa} \int d S \cos q z \cos n \theta E_{z}^{(e)}(a, \theta, z) \\
= & -\frac{q n \psi_{\theta}}{16 \pi^{3} a^{2}} \frac{Z_{0} I_{0}}{\kappa} .
\end{aligned}
$$

Using the following relation

$$
\begin{aligned}
Z_{0}\left(H_{\theta-}-H_{\theta+}\right)= & \frac{Z_{0} I_{0}}{2 \pi a}-j k a \sum_{n} \int d q A_{n}^{(e)} P_{n}(q) \\
& -\sum_{n} \int d q \frac{q n}{\kappa} B_{n}^{(e)} Q_{n}(q)
\end{aligned}
$$

for the even part of the impedance we obtain

$$
\frac{Z^{(e)}}{Z_{0}}=\frac{j k}{8 \pi^{2} a^{2}} \psi_{\theta}\left(1-\frac{\psi_{\theta}}{8 \pi^{2}} V\right),
$$

where $V$ is defined as

$$
V=\sum_{n}\left\{\int d q\left[k^{2} P_{n}(q)-\frac{n^{2} q^{2}}{\kappa^{2} a^{2}} Q_{n}(q)\right]\right\} .
$$

As before, for $\psi_{\theta} V \ll 1$ we can write

$$
Z_{0} Y_{\|}^{(e)}=-\frac{j 8 \pi^{2} a^{2}}{k \psi_{\theta}}-\frac{j a^{2}}{k} V .
$$

We can also combine Eqs. (18) and (25) to obtain the expression for the total impedance

$$
\frac{Z_{\|}}{Z_{0}}=\frac{j k a}{8 \pi^{2} a^{3}}\left[\psi-\chi-\frac{1}{8 \pi^{2}}\left(\psi^{2} V+\chi^{2} W\right)\right] .
$$

Below the pipe cutoffs one readily gets the expression for $\operatorname{Re}(Z)$, already reported in [2,3], corresponding to the TEM mode $[n=0, q=k$ in Eqs. (19) and (26)]. The structure obtained in Eq. (28) is similar to the one presented in [4], but we now have additional contribution from the modes in the coaxial region.

\section{Discussion}

We now remind the reader of the expression for the admittance of an azimuthally symmetric pillbox, obtained only for the magnetic ("even" in our notation) portion of the problem [1]:

$$
\begin{aligned}
Z_{0} Y_{\|}=2 \pi k a & {\left[-\frac{j}{k^{2} g(b-a)}\right.} \\
& \left.+\sum_{s=1}^{\infty} \frac{e^{-j b_{s} g / a}}{b_{s}}+j \frac{2 \ln 2}{\pi}\right],
\end{aligned}
$$

where the pillbox of outer radius $b$ extends from $z=$ 0 to $z=g$ along the axis of the pipe, with $k g \ll 1$, $k(b-a) \ll 1, a$ being the radius of the pipe, and $b_{s}^{2}=$ $k^{2} a^{2}-p_{s}^{2}$ with $p_{s}$ being the roots of $J_{0}\left(p_{s}\right)=0$. One sees in the above expression that the real part of the admittance is independent of all features of the pillbox for $g \ll a$. It can be shown that this term corresponds to the energy which is lost as the pillbox generates outgoing propagating modes in the pipe. Apparently the reactive part arises from the evanescent pipe modes generated by the pillbox.

We now examine our results in Eqs. (20) and (27). One sees that each admittance separates into a part which includes the geometry of the hole (parameters $\psi$ and $\chi$ ) and the term involving the pipe and the coaxial region. In the present case, the real part includes dependence on parameters $b$ and $a$ (where $b$ is the radius of the outer pipe) and is present even below all possible cutoffs because of the existence of the TEM mode in the coaxial region. Additional energy is lost when other outgoing propagating modes are generated in the coaxial and the pipe regions. In fact, experience with Eq. (29) suggests that these expressions are valid in the region of the mode cutoffs where $k a$ and $k(b-a)$ are of the order of 1 . This speculation is confirmed in the numerical studies that follow. 


\section{Numerical Implementation}

It is clear from Eq. (28) that the departure from the usual small hole (Bethe) approximation is contained in the quantities $W$ and $V$ defined in Eqs. (19) and (26). Evaluation of these quantities analytically presents a major challenge requiring some form of cutoffs for large $q$ and large $n$. We assume these cutoffs make $W$ and $V$ finite and proceed to explore their values numerically.

To understand the frequency dependence of the quantities $V$ and $W$, we rewrite expressions (27) and (20) as

$$
\frac{k Z_{0}}{Z^{(e)}}+\left.\frac{Z_{0}}{Z^{(e)}}\right|_{k \rightarrow 0}=-j V a^{2}
$$

and

$$
\frac{k Z_{0}}{Z^{(o)}}-\left.\frac{Z_{0}}{Z^{(o)}}\right|_{k \rightarrow 0}=-j W a^{2} .
$$

We then use the notation $k Z_{0} / Z=k(G+j B)$ and $W=$ $W_{r}+W_{i}, V=V_{r}+V_{i}$. The real parts $W_{r}, V_{r}$ contribute to the imaginary part of the impedance. We use the computer program developed to calculate the impedance of a rectangular hole to obtain data for small holes of different dimensions. In Figs. 1 and 2 we plot $Z_{0}^{-1} k B$ vs $k^{2} a^{2}$ for hole dimensions $w / a=l / a=0.25$ with $a=16[\mathrm{~mm}]$ (where $w$ is the width and $l$ is the length of the hole) for the odd and even parts, respectively. We find linear dependence for both the odd and even parts. We therefore conclude that for low frequencies $V_{r}, W_{r} \sim k^{2}$. Note that in the limit of low frequencies $k^{2}$ deviation from the constant value, which corresponds to the static approximation, is very small. Similar results were obtained for $l / a=0.15,0.35$, and 0.5 with $w / a=0.25$, and $l / a=0.125,0.15,0.25,0.35$, and 0.5 with $w / a=0.125$. For the real part of the admittance in the limit of low frequency, we find that for both the odd and even parts $G=11.55$, which is $\pi /[\ln (b / a)]$ for the value $b / a=1.3125$ we used in the calculations. We test

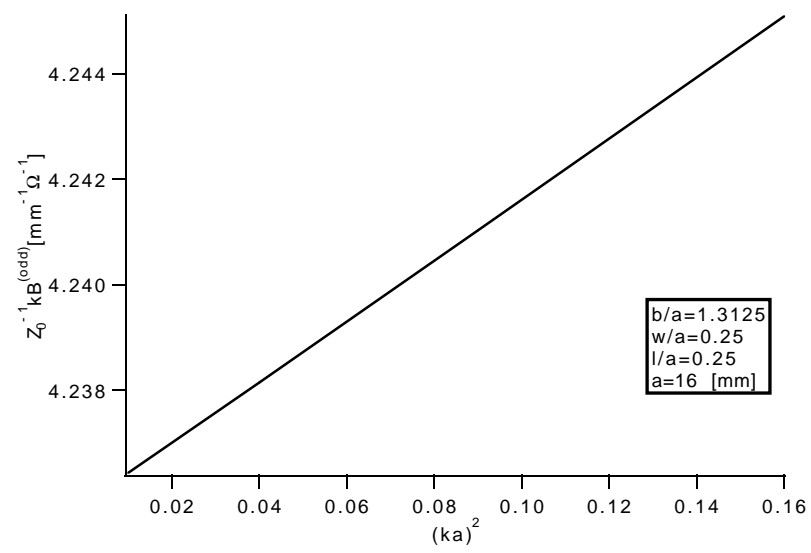

FIG. 1. Electric part of the imaginary admittance at low frequencies. this number for different frequencies from $k a=0.1$ to $k a=0.5$ and hole length $l / a=0.15,0.25,0.35$, and 0.5 with $w / a=0.25$, and find that to several significant figures this number is independent of the frequency and hole dimensions, corresponding to the TEM mode in the coaxial region. We then perform a similar calculation for $b / a=1.2$ to obtain $G=17.23$, which is again independent of frequency and hole dimensions, and agrees with $\pi /[\ln (b / a)]$. We therefore conclude that below all possible cutoffs $V_{r}, W_{r}=\pi /[\ln (b / a)]$, with additional energy being lost when other outgoing propagating modes are generated.

To compare the frequency behavior of the admittance of a small hole with the one presented for a pillbox [1], we perform numerical calculations for the square hole with the following parameters: $b / a=1.3125, w / a=0.25$. Results are shown in Figs. 3 and 4 for the imaginary and real parts of the admittance, respectively. Figure 4 clearly shows the steps which occur as $k a$ passes the cutoffs corresponding to the TM amd TE modes. For the odd part one sees the cutoffs corresponding to the $\mathrm{TE}_{11}^{\text {(coax.) }}, \mathrm{TE}_{21}^{\text {(coax.) }}, \mathrm{TE}_{11}^{\text {(pipe) }}$, and $\mathrm{TE}_{31}^{\text {(coax.) }}$ modes, while for the even part one sees the cutoff corresponding to the $\mathrm{TM}_{01}^{(\mathrm{pipe})}$ mode. This leads to the conclusion that, for a small hole, the odd (electric) part primarily couples to the TE modes, while the even (magnetic) part primarily couples to the TM modes near the cutoffs. This behavior can also be shown analytically (see for example [5]). We also confirmed numerically that this behavior holds for slots with $l / a<1$ and $k l<1$, in agreement with [5]. If one goes to very high frequency $(k l \sim \pi)$, the imaginary part of the admittance in Fig. 3 eventually crosses the zero axis and changes sign. This behavior corresponds to a resonance due to the hole length. Note that the resonances occur at different frequencies for the odd and even parts. The detailed study of resonances related to the slot length can be found in [3].

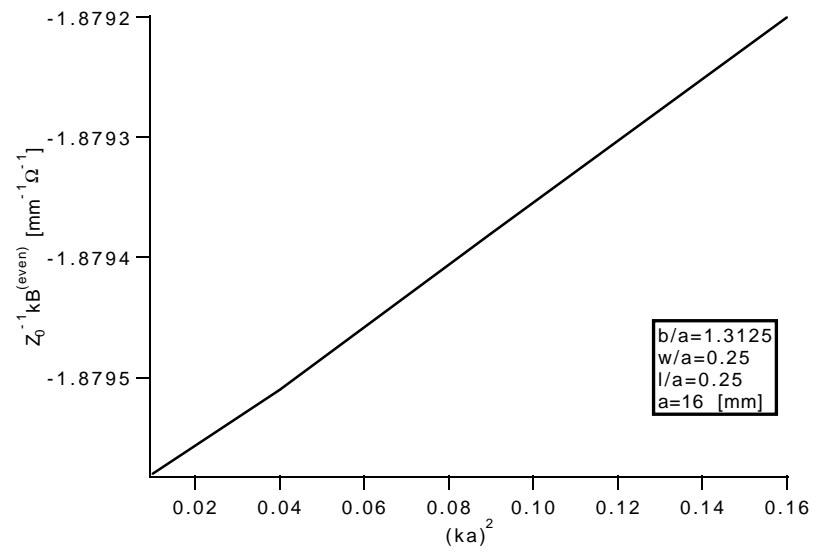

FIG. 2. Magnetic part of the imaginary admittance at low frequencies. 

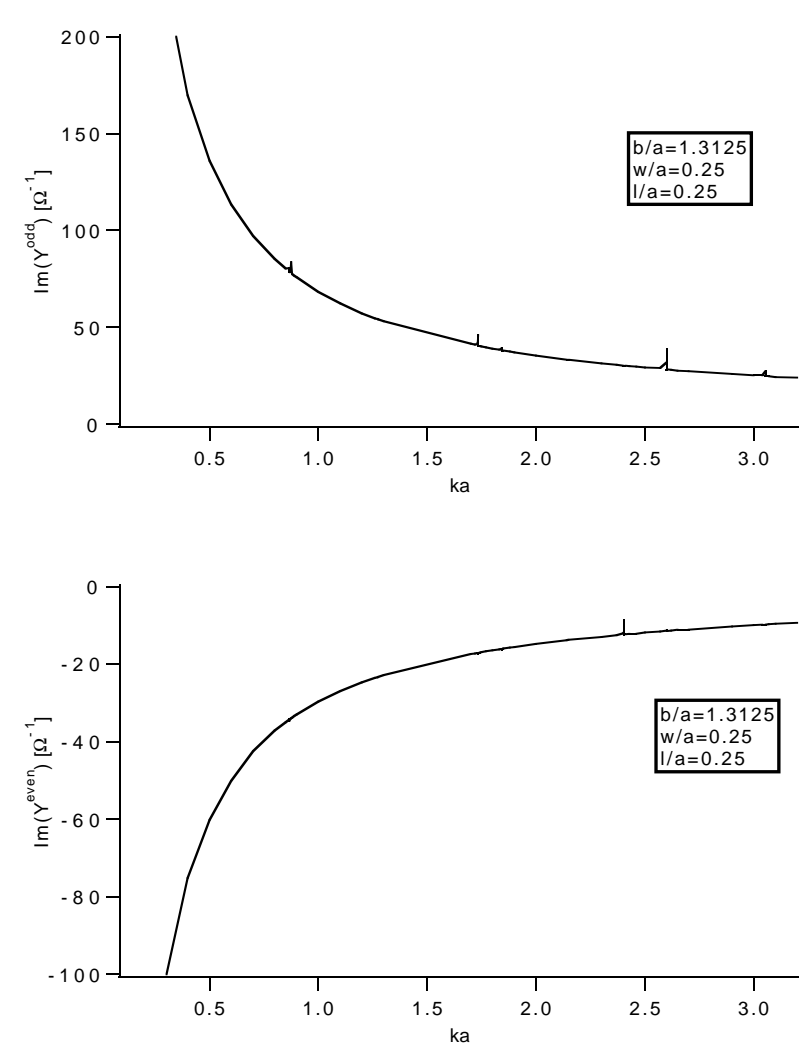

FIG. 3. Imaginary part of the admittance near the cutoff frequencies.

\section{THICK WALL ANALYSIS}

When the field is incident on a hole in a wall of finite thickness, the usual approach is to split it into two components: one with an asymmetric potential and one with a symmetric potential about the midpoint of the wall [6]. The parameters $\psi$ and $\chi$ come from both the symmetric and antisymmetric problem. We then define the magnetic susceptibility and electric polarizability seen within the liner as

$$
\begin{aligned}
& \psi_{\text {in }}=\psi^{s}+\psi^{a}, \\
& \chi_{\text {in }}=\chi^{s}+\chi^{a},
\end{aligned}
$$

while the susceptibility and polarizability outside the liner are defined by

$$
\begin{aligned}
& \psi_{\text {out }}=\psi^{s}-\psi^{a}, \\
& \chi_{\text {out }}=\chi^{s}-\chi^{a} .
\end{aligned}
$$

For the wall of zero thickness $\psi^{a}=\chi^{a}=0$, making $\psi_{\text {in }}=\psi^{s}, \chi_{\text {in }}=\chi^{s}$.

\section{A. Even part}

We again treat the problem as quasistatic in the hole region where $E_{r}, H_{\theta}$ are denoted by $E_{1}, H_{1}$ near the inside
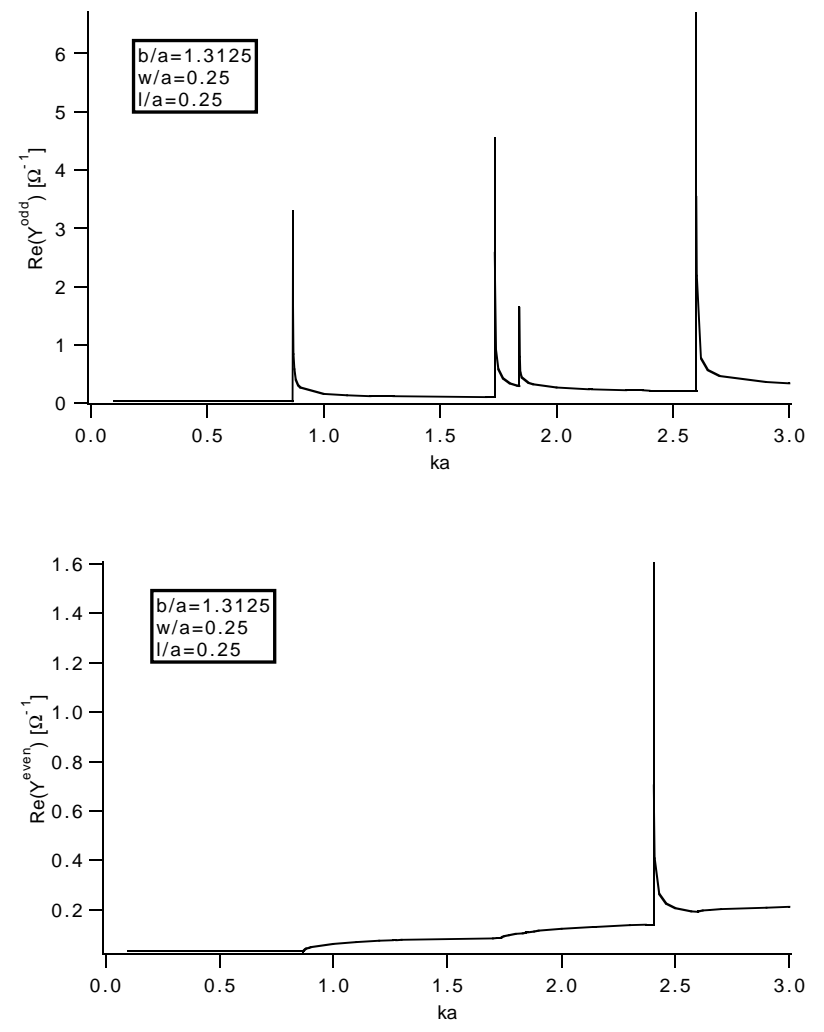

FIG. 4. Real part of the admittance near the cutoff frequencies.

surface of the hole and by $E_{2}, H_{2}$ near the outside surface. For the even part of the impedance we have

$$
\frac{Z^{(e)}}{Z_{0}}=-\frac{1}{2 \pi a Z_{0} I_{0}} \int d S E_{z}^{(e)}(a, \theta, z) \cos k z,
$$

which can be rewritten as

$$
\frac{Z^{(e)}}{Z_{0}}=-\frac{j k Z_{0}}{2 \pi a Z_{0} I_{0}}\left(\frac{H_{1}}{2} \psi_{\text {in }}-\frac{H_{2}}{2} \psi_{\text {out }}\right) .
$$

Using the following expression

$$
\int_{r=a} d S E_{z}(a, \theta, z)=-\frac{j k Z_{0}}{2} H_{1} \psi_{\text {in }}
$$

and performing field matching at the inner surface of the liner, we obtain the expansion coefficients in the pipe region

$$
\begin{aligned}
& A_{n}^{(e)}=-\frac{j k Z_{0} H_{1}}{8 \pi^{2} a} \psi_{\text {in }}, \\
& B_{n}^{(e)}=-\frac{q n Z_{0} H_{1}}{8 \pi^{2} a^{2} \kappa} \psi_{\text {in }} .
\end{aligned}
$$

In the approximation of a small hole for $H_{1}$ at the inner surface of the liner, we then have 


$$
\begin{aligned}
Z_{0} H_{1} & =\frac{Z_{0} I_{0}}{2 \pi a}-j k a \sum_{n} \int d q A_{n}^{(e)} \frac{J_{n}^{\prime}(\kappa a)}{\kappa a J_{n}(\kappa a)}-\sum_{n} \int d q \frac{q n}{\kappa} B_{n}^{(e)} \frac{J_{n}(\kappa a)}{\kappa a J_{n}^{\prime}(\kappa a)} \\
& =\frac{Z_{0} I_{0}}{2 \pi a}\left\{1-\frac{1}{8 \pi^{2} a} \psi_{\text {in }} \sum_{n} \int d q\left[\frac{k^{2} J_{n}^{\prime}(\kappa a)}{\kappa J_{n}(\kappa a)}-\frac{q^{2} n^{2}}{\kappa^{2} a^{2}} \frac{J_{n}(\kappa a)}{\kappa J_{n}^{\prime}(\kappa a)}\right]\right\} .
\end{aligned}
$$

Using

$$
\int_{r=d} d S E_{z}(d, \theta, z)=-\frac{j k Z_{0}}{2} H_{1} \psi_{\mathrm{out}},
$$

for the $\mathrm{H}_{2}$ at the outer surface of the liner, we similarly obtain

$$
Z_{0} H_{2}=-\frac{Z_{0} I_{0}}{2 \pi a} \frac{1}{8 \pi^{2} d} \psi_{\text {out }} \sum_{n} \int d q\left[\frac{k^{2} F_{n}^{\prime}(\kappa d)}{\kappa F_{n}(\kappa d)}-\frac{q^{2} n^{2}}{\kappa^{2} d^{2}} \frac{G_{n}(\kappa d)}{\kappa G_{n}^{\prime}(\kappa d)}\right],
$$

where $r=d$ is the outer surface of the liner. Setting $a=d$, except for the parameters $\psi_{\text {in }}$, $\psi_{\text {out }}$, we obtain the expression for the even part of the impedance

$$
\frac{Z^{(e)}}{Z_{0}}=\frac{j k}{8 \pi^{2} a^{2}} \psi_{\text {in }}\left(1-\frac{\psi_{\text {in }}}{8 \pi^{2}} \tilde{V}\right)
$$

where

$$
\tilde{V}=\sum_{n} \int d q\left\{k^{2}\left[\frac{J_{n}^{\prime}(\kappa a)}{\kappa a J_{n}(\kappa a)}-\frac{\psi_{\mathrm{out}}^{2}}{\psi_{\mathrm{in}}^{2}} \frac{F_{n}^{\prime}(\kappa a)}{\kappa a F_{n}(\kappa a)}\right]-\frac{q^{2} n^{2}}{\kappa^{2} a^{2}}\left[\frac{J_{n}(\kappa a)}{\kappa a J_{n}^{\prime}(\kappa a)}-\frac{\psi_{\mathrm{out}}^{2}}{\psi_{\mathrm{in}}^{2}} \frac{G_{n}(\kappa a)}{\kappa a G_{n}^{\prime}(\kappa a)}\right]\right\} .
$$

For the odd part of the impedance we have

\section{B. Odd part}

$$
\frac{Z^{(o)}}{Z_{0}}=-\frac{j}{2 \pi a Z_{0} I_{0}} \int d S E_{z}^{(o)}(a, \theta, z) \sin k z=-\frac{j k}{2 \pi a Z_{0} I_{0}} \int_{r=a} \Phi d S .
$$

In the above expression the integral can be rewritten in the following form:

$$
\int_{r=a} \Phi d S=E_{1} \frac{\chi_{\text {in }}}{2}-E_{2} \frac{\chi_{\text {out }}}{2} .
$$

For the radial component of the electric field $E_{1}$ on the inner surface of the liner, we have the following expansion coefficients:

$$
\begin{aligned}
A_{n}^{(o)} & =\frac{Z_{0} I_{0}}{16 \pi^{3} a^{2}} j q \chi_{\mathrm{in}}, \\
B_{n}^{(o)} & =\frac{Z_{0} I_{0}}{16 \pi^{3} a^{2}} \frac{n k}{\kappa a} \chi_{\mathrm{in}} .
\end{aligned}
$$

We then obtain

$$
E_{1}=\frac{Z_{0} I_{0}}{2 \pi a}\left\{1+\frac{\chi_{\text {in }}}{8 \pi^{2}} \sum_{n} \int d q\left[\frac{q^{2} J_{n}^{\prime}(\kappa a)}{\kappa a J_{n}(\kappa a)}-\frac{n^{2} k^{2}}{\kappa^{2} a^{2}} \frac{J_{n}(\kappa a)}{J_{n}^{\prime}(\kappa a)}\right]\right\} .
$$

By performing similar calculations for the electric field $E_{2}$ at the outer surface of the liner, we finally obtain for the odd part of the impedance

$$
\frac{Z^{(o)}}{Z_{0}}=-\frac{j k}{8 \pi^{2} a^{2}} \chi_{\text {in }}\left(1+\frac{\chi_{\text {in }}}{8 \pi^{2}} \tilde{W}\right)
$$

where

$$
\tilde{W}=\sum_{n} \int d q\left\{q^{2}\left[\frac{J_{n}^{\prime}(\kappa a)}{\kappa a J_{n}(\kappa a)}-\frac{\chi_{\mathrm{out}}^{2}}{\chi_{\mathrm{in}}^{2}} \frac{F_{n}^{\prime}(\kappa a)}{\kappa a F_{n}(\kappa a)}\right]-\frac{k^{2} n^{2}}{\kappa^{2} a^{2}}\left[\frac{J_{n}(\kappa a)}{\kappa a J_{n}^{\prime}(\kappa a)}-\frac{\chi_{\mathrm{out}}^{2}}{\chi_{\mathrm{in}}^{2}} \frac{G_{n}(\kappa a)}{\kappa a G_{n}^{\prime}(\kappa a)}\right]\right\} .
$$

Expressions for $\tilde{V}$ and $\tilde{W}$ include the parameters $\psi$ and $\chi$; and, therefore, for the case of a wall of finite thickness, the admittance does not separate into a part which includes only the geometry of a hole and a term which includes the 
geometry of a pipe. But this separation is still valid for a thin wall $\left(\chi_{\text {out }}=\chi_{\text {in }}, \psi_{\text {out }}=\psi_{\text {in }}\right)$ and for a very thick wall $\left(\chi_{\text {out }}=\psi_{\text {out }}=0\right)$.

\section{SUMMARY}

We obtained analytic expressions for the impedance of a small hole which includes effects of energy propagation along the inner beam pipe and/or outer coaxial pipe. This allows us to understand the structure of the impedance in the frequency region near the cutoffs of the inner beam pipe and the outer coaxial structure. Expressions for both the electric and magnetic problems for a hole without azimuthal symmetry were obtained and explored by means of numerical calculations. We then extended our analytic treatment to a wall of finite thickness, discussed the resulting expressions, and concluded that the admittance could no longer be separated into a part depending only on the pipe geometry and a part depending primarily on the hole geometry.

[1] R.L. Gluckstern and F. Neri, in Proceedings of the 1989 Particle Accelerator Conference, Chicago, Illinois (IEEE, New York, 1989), p. 1271.

[2] A. V. Fedotov and R. L. Gluckstern, Phys. Rev. E 56, 3583 (1997).

[3] A. V. Fedotov and R. L. Gluckstern, Phys. Rev. E 56, 7217 (1997).

[4] S. S. Kurennoy, R.L. Gluckstern, and G.V. Stupakov, Phys. Rev. E 52, 4354 (1995).

[5] G. V. Stupakov, in Proceedings of the 1995 Particle Accelerator Conference, Dallas, Texas (IEEE, New York, 1995), p. 3306.

[6] R. L. Gluckstern and J.A. Diamond, IEEE Trans. Microwave Theory Tech. 39, 274 (1991). 